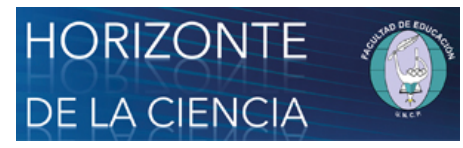

Horizonte de la Ciencia

ISSN: 2413-936X

horizontedelaciencia@uncp.edu.pe

Universidad Nacional del Centro del Perú

Perú

\title{
Estudio etnográfico sobre la comunidad pesquera artesanal de Playa Carpayo (Chucuito), Provincia Constitucional del Callao, Perú
}

Monterroso Coronado, Cesar Antonio; Sánchez Casimiro, Walter Jesús; Quijano Aranibar, Ivan Ernesto; Huamán Sánchez, Miriam Nora

Estudio etnográfico sobre la comunidad pesquera artesanal de Playa Carpayo (Chucuito), Provincia Constitucional del Callao, Perú

Horizonte de la Ciencia, vol. 10, núm. 18, 2020

Universidad Nacional del Centro del Perú, Perú

DOI: https://doi.org/10.26490/uncp.horizonteciencia.2020.18.394

Los autores otorgan el permiso a compartir y usar su trabajo manteniendo la autoría del mismo. Atribución no comercial (CC BY-NC) 4.0 


\section{Estudio etnográfico sobre la comunidad pesquera artesanal de Playa Carpayo (Chucuito), Provincia Constitucional del Callao, Perú}

Ethnographic study on the artisanal fishing community of Playa Carpayo (Chucuito), Provincia Constitucional del Callao, Perú

Estudo etnográfico da comunidade pesqueira artesanal de Playa Carpayo (Chucuito), Província Constitucional de Callao, Perú

Nunakunap yachay makillaan challwa chalaku nunakuna Carpayo harun ucha mayanchu(Chucuito) Callao kamachisha paruwinsia, Piru

\section{IÑANATAKOITATZIRO OKANTAKOTARI NAMPITSIPE SHIMARIANTSINKARO ASHITARI ARORI EMPANEKIITEKI CARPAYO NANPITSIJANIKI PROVINCIA JEBATAKOBENTSIRORI PINKATSARENTSI NAMPITSIPE CALLAO. PERU}

Sangenarentsijenga komantatsatokotagetaigiri ira simaataneriegi pañaigacha Carpayo(chucuito),timagantsi jitacha Ora Kallao, Peru

Cesar Antonio Monterroso Coronado Datos de los autores Escuela Nacional de Marina Mercante "Almirante Miguel Grau", Perú cmonterroso@enamm.edu.pe

(iD http://orcid.org/0000-0001-5407-8741

Walter Jesús Sánchez Casimiro

Escuela Nacional de Marina Mercante "Almirante Miguel

Grau", Perú

wsanchez@enamm.edu.pe

iD http://orcid.org/0000-0002-9404-0120

Ivan Ernesto Quijano Aranibar

Escuela Nacional de Marina Mercante "Almirante Miguel

Grau", Perú

iquijano@enamm.edu.pe
DOI: https://doi.org/10.26490/ uncp.horizonteciencia.2020.18.394

Recepción: 03 Agosto 2019 Aprobación: 06 Octubre 2019

\section{Notas DE AUTOR}

Datos César Antonio Monterroso Coronado. Director de Posgrado. Investigador y docente de metodología de la investigación, métodos cuantitativos y de los macroeconomía. Doctor en Gestión Económica Global por la Universidad Nacional Mayor de San Marcos (UNMSM), Lima Perú. Magister en autores Administración de Negocios Internacionales por la Universidad Católica Sedes Sapientaie (UCSS). Maestría en Finanzas por la Universidad del Pacifico (UP).

Walter Sánchez Casimiro. Jefe de Oficina de Calidad Educativa y Responsabilidad Social. Investigador y docente de metodología de la investigación, estadística aplicada la investigación e investigación operativa. Egresado de Maestría en Educación con mención Gestión de la Educación, en la Universidad Nacional Mayor de San Marcos (UNMSM). Egresado del Doctorado en Ciencias de la Educación, en la UNMSM.

Iván Quijano Aranibar. Docente e investigador. Especialista en metodología cuantitativa, cualitativa y mixta. Licenciado en Educación con mención en Ciencias Histórico Sociales por la Universidad Nacional Federico Villarreal (UNFV). Bachiller en Ciencias Sociales en la especialidad de Arqueología por la Universidad Nacional Mayor de San Marcos (UNMSM). Egresado de la Maestría en Educación con mención en Docencia Universitaria por la UNMSM, Lima Perú.

Miriam Huamán Sánchez. Docente investigador. Investigador y docente de operación y legislación pesquera, ecología y medio ambiente y protección del medio ambiente. Ingeniero Pesquero, por la Universidad Nacional del Callao (UNAC). Egresado de Maestría en Gestión Pesquera por la UNAC. Egresado de Maestría en Gestión Ambiental por la Universidad Nacional Federico Villarreal (UNFV).

cmonterroso@enamm.edu.pe 
http://orcid.org/0000-0003-2264-1186

\author{
Miriam Nora Huamán Sánchez \\ Escuela Nacional de Marina Mercante "Almirante Miguel \\ Grau", Perú \\ mhuaman@enamm.edu.pe \\ (iD http://orcid.org/0000-0001-9327-6337
}

Recepción: 03 Agosto 2019

Aprobación: 06 Octubre 2019

\title{
ReSUMEN:
}

El objetivo de estudio fue conocer el modo de vida de la comunidad pesquera artesanal de Playa Carpayo (Chucuito), Provincia Constitucional del Callao, Perú. El enfoque de investigación es cualitativo y el tipo no experimental. Se ha elegido el diseño etnográfico de corte transversal y se ha tenido acceso a 2 pescadores artesanales de mencionada comunidad (PAC-A y PACB). Asimismo, se aplicó la entrevista semiestructurada, a través del instrumento ENAMM-PAC1 constituido con 26 preguntas abiertas (ítems 1-26). Los resultados preliminares demuestran que los pescadores artesanales de Playa Carpayo comparten rasgos generales con otras comunidades de pescadores, pero a su vez contemplan ciertas variaciones como se aprecia en su constitución e historia.

Palabras Clave: Chucuito, etnografía, Perú, pescador artesanal, Playa Carpayo, Chucuito, nunalisina, Piru, makip challwachalaku, Carpayo ucha manya, nanpitsijaniki, ñanatakotsirori nampitsipe, peru, shimariantsi ashitari arori empanekiite carpayo, timagantsigite, sangenatëgotagetiro kibatsi, matagiteripage, obarage.

\section{Abstract:}

The objective of the study was to know the way of life of the artisanal fishing community of Playa Carpayo (Chucuito), Constitutional Province of Callao, Peru. The research approach is qualitative and the type is not experimental. The cross-type ethnographic design has been chosen. It has had access to 2 artisanal fishermen from that community (PAC-A and PAC-B). The semi-structured interview was applied through the ENAMM-PAC1 instrument consisting of 26 open questions (items 1-26). Preliminary results show that the artisanal fishermen of Playa Carpayo have general characteristics with other fishing communities, but in turn contemplate certain variations as can be seen in their constitution and history.

KEYWORDS: artisanal fisherman, Carpayo beach, Chucuito, ethnography, Perú.

\section{Resumo:}

O objetivo do estudo foi conhecer o modo de vida da comunidade de pescadores artesanais de Playa Carpayo (Chucuito), Provincia constitucional de Callao, Peru. A abordagem da pesquisa é qualitativa e o tipo não experimental. O desenho etnográfico transversal foi escolhido e dois pescadores artesanais dessa comunidade foram acessados (PAC-A e PAC-B). Da mesma forma, foi aplicada a entrevista semiestruturada, por meio do instrumento ENAMM-PAC1 constituído por 26 questões abertas (itens 1-26). Resultados preliminares mostram que os pescadores artesanais de Playa Carpayo compartilham características gerais com outras comunidades de pescadores, mas, por sua vez, contemplam certas variações, como podem ser vistas em sua constituição e história. Palavras-Chave: Chucuito, etnografia, Peru, pescador artesanal, Praia de Carpayo.

\section{INTRODUCCIÓN}

La pesca artesanal es una actividad imprescindible en la economía nacional de las regiones costeñas del Perú, ya que fomenta el empleo y el suministro de recursos hidrobiológicos a la población local (Agencia Española de Cooperación Internacional, 2003). Esto se debe a que el mar peruano ofrece una mayor diversidad y variabilidad de recursos que posibilitan y dinamizan la cadena alimenticia marina. Producto de ello, se concentran poblados de pescadores a pequeña escala o artesanales dispersos por todo el litoral, principalmente por el norte desde Piura hasta Áncash y por el sur desde Ica hasta Moquegua (Medicina, 2014). 
En este marco, la Ley General de la Pesca N²5977 (21 de diciembre de 1992), en su Artículo 20, entiende por pescador artesanal aquella persona, natural o jurídica, que realiza la captura de los recursos hidrobiológicos mediante el predominio del trabajo manual, con o sin embarcación pequeñas de hasta 15 metros de eslora (longitud de proa a popa); en oposición a la pesca industrial o de mayor escala. No obstante, McGoodwin (2002) sostiene que el término artesanal es subjetivo y ambiguo; por lo que sería apropiada la categoría de pescador a pequeña escala, resultado de la reducida envergadura de sus inversiones de capital y niveles de producción. Frente a esta dicotomía (pescador artesanal vs. pescador a pequeña escala), el Ministerio de Producción, diferencia al pescador artesanal del pescador a menor escala, debido a que carece de modernos equipos para la pesca (Decreto Supremo N012, 14 de marzo de 2001) y sus embarcaciones tienen un máximo de 10 metros cúbicos de capacidad de bodega. Inclusive, su zona de explotación de recursos se circunscribe entre las 0 y 5 millas (Decreto Supremo N 005,25 de agosto de 2012). Por consiguiente, se considera que las definiciones establecidas por la Ley General de la Pesca N²5977 (21 de diciembre de 1992), y sus respectivos Decretos Supremos (D.S. N012, 14 de marzo de 2001; D.S. N005, 25 de agosto de 2012), y por McGoodwin (2002) no son excluyentes, puesto que, al hacer referencia a los pescadores artesanales, también se está tomando en cuenta los bajos niveles de producción y de capital, sea el caso de los $10 \mathrm{~m} 3$ de volumen de almacenaje, las técnicas convencionales, las 5 millas etc., dado que se busca asegurar la sostenibilidad de los ecosistemas acuáticos gracias a la explotación racional (Medicina, 2014).

Por su parte, Neira (2005), siguiendo a MacGoodwin (2002), al referirse a los pescadores artesanales, adiciona la dimensión identitaria, pues afirma que formar parte de ésta comunidad permite participar de lazos sociales, políticos, culturales e ideológicos construidos por un grupo humano cohesionado.

Desde luego, en el Perú se han realizado un limitado número de investigaciones descriptivas sobre los pescadores artesanales. Entre ellas, destaca el diagnóstico de Galarza y Kámiche (2015) para caracterizar y analizar esta actividad de subsistencia. En líneas generales, ambos autores sostienen que la cadena de valor de la pesca artesanal está compuesta por 4 fases: extracción, procesamiento, comercialización y consumo final. Asimismo, los pescadores artesanales inician este proceso de producción, pero son los que menos beneficios económicos reciben, debido a que tienen un elevado nivel de necesidades básicas insatisfechas. De igual modo, respecto a las embarcaciones, dan poca importancia a la titularidad en la inscripción de registros públicos y el $28 \%$ carece de un sistema de preservación de recursos pesquero; por lo que afecta a la calidad de la mercancía final. Encima, más del $85 \%$ de pescadores artesanales financian su faena de pesca (capital de trabajo), a través de los recursos emitidos por el comerciante (intermediario) y, por último, la falta de cámaras de conservación y bloques de hielo son factores que impulsan al pescador a vender productos de menor valor agregado. De la misma manera, sobresale el diagnóstico socio-económico de los pescadores artesanales de Ilo y Tacna elaborado por la Agencia Española de Cooperación Internacional. Entonces, AECI (2003) resalta la conformación de los pescadores artesanales constituidos principalmente por migrantes, específicamente de la región andina, y la inserción de sus familiares, sea el caso de sus hijos, pues se aprecian pescadores más jóvenes. Sin embargo, el grueso de ellos no ha cumplido la Educación Básica Regular, pero existe un grupo minoritario con nivel educativo Superior. Por ello, Galarza y Kámiche (2015) sostienen que el nivel educativo está correlacionado positivamente con los ingresos de faena de pesca; por lo que a mayores estudios, existe un mayor margen de ganancia y viceversa. Por otro lado, AECI (2003) también resalta la percepción de los pescadores en relación a las malas prácticas sobre los recursos hidrobiológicos, cuyo principal factor lo atribuyen a la ausencia de control y/o fiscalización que intensifica la depredación de los recursos marítimos, vale decir, una actividad supuestamente sostenible se torna en irracional. Además, como punto axial, sus resultados enfatizan la sensibilización de la comunidad de pescadores tradicionales sobre esta praxis y sus repercusiones.

Antes este escenario, a pocos metros de la Escuela Nacional de Marina Mercante (ENAMM), se encuentran los pescadores artesanales de Playa Carpayo (Chucuito), Provincia Constitucional del Callo, Perú, una comunidad de pescadores tradicionales desconocidos en la literatura existente sobre esta temática, 
tanto a nivel nacional e internacional. En consecuencia, el presente trabajo, de naturaleza preliminar, va a contribuir a enriquecer el corpus teórico sobre los pescadores artesanales y, paulatinamente, develar el modo de vida de este grupo humano. Igualmente, dará inicio a una investigación diacrónica sobre Responsabilidad Social Universitaria (RSU) en la comunidad educativa de ENAMM, dado que en futuras investigaciones se buscará generar un impacto social en la sociedad local y fomentar el desarrollo socio-económico en los pescadores colindantes, según los principios de la RSU establecidos por Vallaeys (2016).

En suma, tomando estas ideas de cabecera, se tiene el siguiente objetivo general: conocer el modo de vida de la comunidad pesquera artesanal de Playa Carpayo (Chucuito), Provincia Constitucional del Callao, Perú. Por ende, se han propuesto los siguientes objetivos específicos:

- Conocer la historia de la comunidad de pescadores artesanales de Playa Carpayo-Chucuito.

- Describir el aspecto social de la comunidad de pescadores artesanales de Playa Carpayo-Chucuito.

- Reconocer la perspectiva económica de la comunidad de pescadores artesanales de Playa CarpayoChucuito.

- Conocer la conciencia ambiental de la comunidad de pescadores artesanales de Playa Carpayo-Chucuito.

- Precisar el nivel educativo de la comunidad de pescadores artesanales de Playa Carpayo-Chucuito.

- Conocer el marco ideológico de la comunidad de pescadores artesanales de Playa Carpayo-Chucuito.

\section{Metodología}

La investigación es de enfoque cualitativo y de tipo no experimental. Se empleó el diseño de investigación etnográfico de corte transversal, puesto que se realizará una descripción e interpretación de un grupo humano en un tiempo y un contexto dado (Álvarez-Gayou, 2009), es decir se conocerá el capital de una comunidad pesquera artesanal de Chucuito, Provincia Constitucional del Callao, Perú en el 2019.

La población está constituida por 8 individuos de la comunidad de pescadores artesanales de Playa Carpayo, Chucuito, Provincia Constitucional del Callao, Perú (Figura 1), asentados a 200 metros aproximadamente de la Escuela Nacional de Marina Mercante. Por su parte, como nos situamos dentro de un estudio de naturaleza cualitativa, la muestra es prescindible en términos de representatividad (ÁlvarezGayou, 2009). Por tanto, se ha tenido acceso a 2 pescadores artesanales de mencionada comunidad (PAC-A y PAC-B), dado que es un sector reacio e intolerante a la participación y diálogo sobre su faena.

La técnica seleccionada ha sido la entrevista, puesto que es un procedimiento que permite registrar información empírica presente en el relato de las personas y/o actores sociales situados en un contexto histórico particular (Cruz, 2007). Se ha seleccionado la clase de entrevista semiestructurada, debido a que se ha abordado con cierto grado de flexibilidad a los participantes de la comunidad de pescadores artesanales de Playa Carpayo, a partir de un conjunto de preguntas previamente establecidas, con la finalidad de generar confianza en ellos y colectar información fidedigna. Por esta razón, se ha elaborado una guía de entrevista denominada ENAMM-PAC1 con 26 preguntas abiertas (ítems 1-26), ver tabla 1. 
TABLA 1

Ítems del instrumento ENAMM-PAC1

\begin{tabular}{|c|}
\hline Preguntas de la guía de \\
\hline 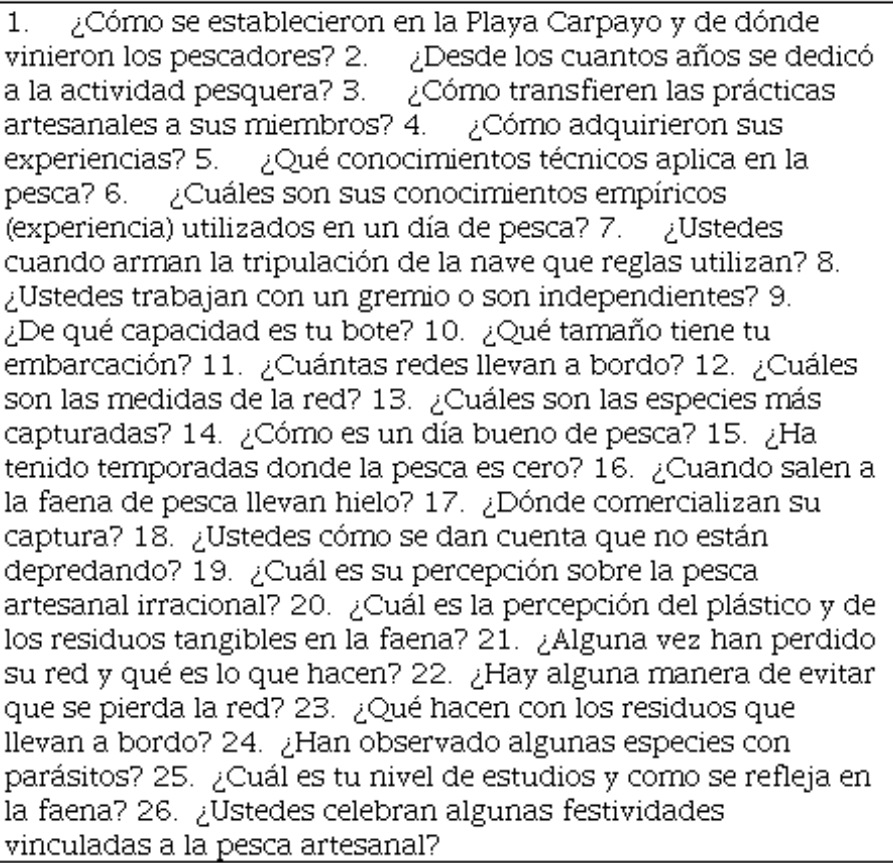 \\
\hline
\end{tabular}

Fuente: elaboración propia

El instrumento ENAMM-PAC1 ha sido elaborado mediante la categoría pescador artesanal de Playa Carapayo y sus respectivas sub-categorías. Además, cada una de éstas, para un registro minucioso, presenta un conjunto de características. Todo ello, se contemplan en la tabla 2. 
TABLA 2

Categoría (C) y sub-categorías $(\mathrm{Sc})$ e instrumento (I) con sus respectivos ítems

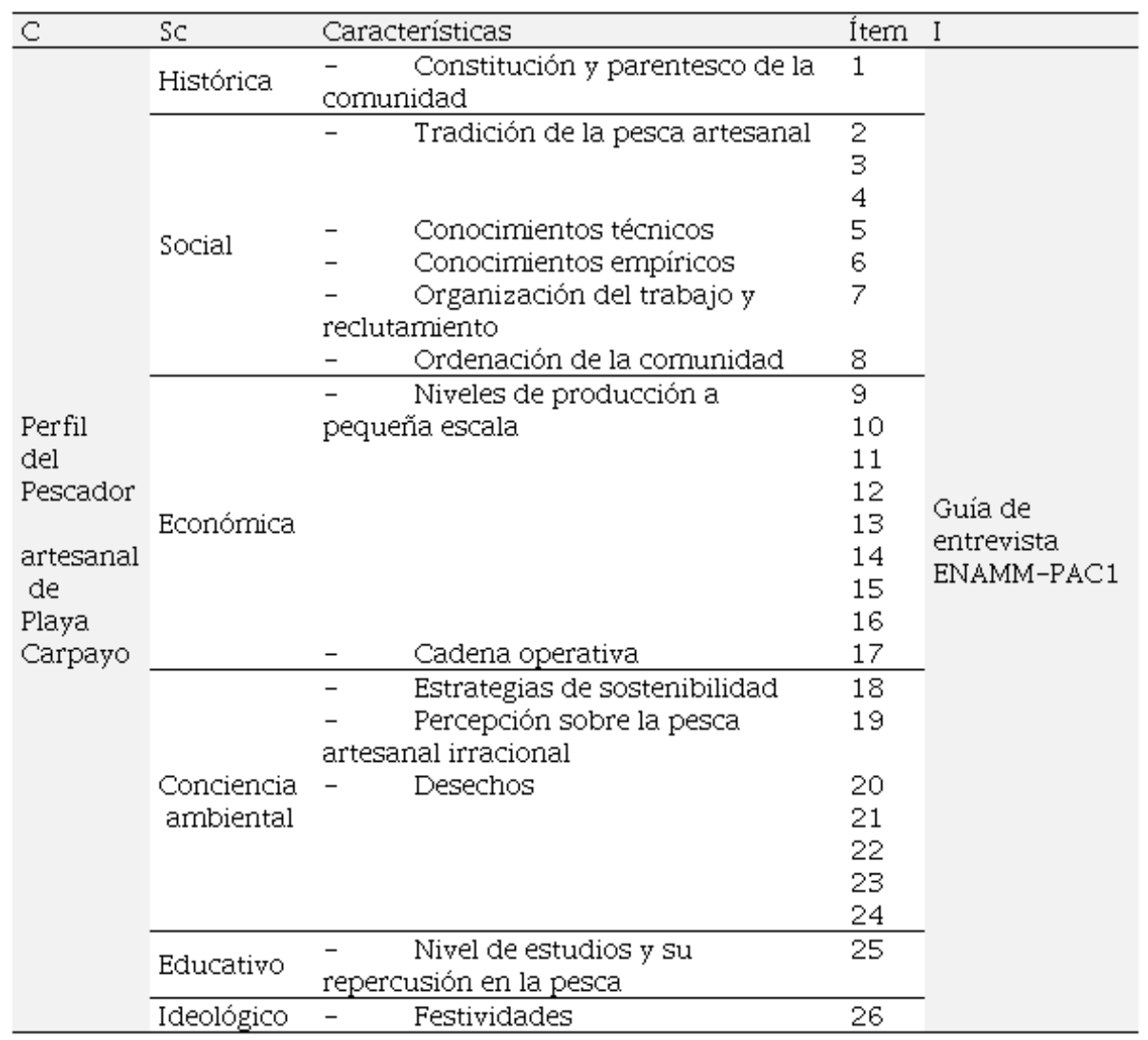

Fuente: elaboración propia

\section{Resultados}

La data empírica recolectada por la aplicación del instrumento ENAMM-PAC1, a partir de la entrevista semiestructurada a los 2 pescadores artesanales de Playa Carpayo (PAC-A y PAC-B), ha sido registrada por medio de una grabadora, con pleno conocimiento y autorización de ambos, para luego ser transcrita (Apéndice 1) y codificada en la siguiente matriz a base de las sub-categorías y sus respectivas características de estudio, ver tabla 3. 
TABLA 3

Codificación de los audios a partir de las sub-categorías $(\mathrm{Sc})$ y características $(\mathrm{C})$

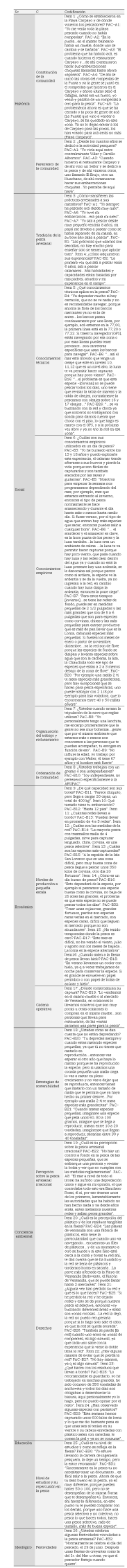


Fuente: elaboración propia.

\section{DiscuSIÓN}

En cuanto a la discusión, según Medicina (2014) los pescadores artesanales están dispersos por todo el litoral peruano atraídos por la variabilidad de recursos marítimos; por lo que los pescadores tradicionales de Playa Carpayo (Chucuito), no son la excepción. Estos, explotan los recursos hidrobiológicos dentro de las 5 millas, límite establecido por el Ministerio de Producción (D.S. N005, 25 de agosto de 2012), pero en ocasiones infringen esta normativa. Esto se infiere por la pesca inusual de la lisa voladora, cuyo nicho oscila entre las 40 y 50 millas (PAC-B20). Asimismo, las embarcaciones tienen de eslora 3,5 metros aproximadamente (PACB12), longitud permitida por la Ley General de la Pesca N²5977 (21 de diciembre de 1992), y su capacidad de carga es de 400 kilogramos (PAC-B1), en contraste con lo establecido por el Estado (D.S. N005, 25 de agosto de 2012). Además, si bien es cierto que una condición es la ausencia de modernos equipos (D.S. N ○012, 14 de marzo de 2001), usan, como herramienta de georreferenciación, el GPS para evitar las carreteras específicas de navegación de los barcos mercantes (PAC-B4) y para impedir que las redes se atasquen y se pierdan en un terreno agreste (PAC-B25). Por todo lo mencionado, la comunidad de pescadores de Playa Carpayo cuenta con un nivel bajo de capital y producción, como ha sido establecido por McGoodwin (2002).

Respecto a la subcategoría histórica y social, los resultados son similares a los establecidos por AECI (2003), dado que los pescadores artesanales de Playa Carpayo se han constituido a partir de migrantes, específicamente del Huachano (PAC-A3), sin dejar de mencionar la contribución local (PAC-A1) y, de igual manera, buscan que sus descendientes continúen con la tradición artesanal, caso del PAC-A quien menciona que contaba con una embarcación para su nieto (PAC-A6).

En relación a la sub-categoría económica, se han alcanzado resultados equivalentes a los sostenidos por Galarza y Kámiche (2015) sobre la financiación de la faena y la titularidad de las embarcaciones para la pesca. Esto es debido a que los pescadores tradicionales de Carpayo financian su propia actividad pesquera producto de las ventas en los muelles y mercados (PAC-B19) y no le dan importancia a la titularidad en la inscripción de registros, pues, no figura en la Asociación regional Unión de Pescadores Artesanales y Conexos del Callao (ARUPAC) (PAC-B10). De igual forma, en concordancia con ambos autores, las comunidades de pescadores inician la cadena operativa de la pesca artesanal, gracias a la fase extractiva, pero usan de manera limitada el hielo para la preservación de los recursos pesqueros, dado que los pescadores de Carpayo lo utilizan estacionalmente en verano (PAC-B18), generando bajos ingresos económicos, a diferencia de los intermediarios que cuentan con bodegas y cámaras de refrigeración. A pesar de ello, abastecen a la población local, de manera directa e indirecta (PAC-B19), como ha sido afirmado por AECI (2003).

Con respecto a la sub-categoría conciencia ambiental, AECI (2003) tempranamente puntualizo la percepción de los pescadores sobre las malas prácticas de explotación de recursos hidrobiológicos, esto se expresa, según la comunidad pesquera de Carpayo, a través de la utilización inadecuada de redes ciegas que no permiten la reproducción de los peces o al menos de su primer desove (PAC-B20) y del atascamiento de las redes en el fondo del mar por las zonas rocosas, pero esta última la atribuyen como una pericia natural y normal (PAC-B25; PAC-B26; PAC-B27). También, comentan sobre la contaminación de las playas producto de los residuos de plástico en el mar (PAC-B24) y recomiendan guardar y desembarcar en tierra los residuos generados en la faena (PAC-B28). Todo esto, como asevera AECI (2003), atribuyen, como factor axial, a la ausencia de control y fiscalización de las autoridades sobre la actividad pesquera artesanal (PAC-B22; PAC-A8).

En función al nivel educativo, los resultados son equiparables a los sostenidos por AECI (2003), debido a que la mayoría no ha cumplido la Educación Básica Regular, pero existe un grupo minoritario con nivel Superior, esto se ve reflejado en el PAC-B que ha retomado la carrera de ingeniería pesquera (PAC-B30). A pesar que Galarza y Kámiche (2015) afirman que el nivel educativo es directamente proporcional al nivel 
educativo, como se manifiesta en el PAC-B con estudios no concluidos de Pregrado, pero éste considera que sus habilidades y capacidades para la pesca están sustentadas en su experiencia y aprendizaje intergeneracional (PAC-B2).

Por último, respecto a la sub-categoría ideológica, siguiendo a Neira (2005), los pescadores de Playa Carpayo comparten un conjunto actividades culturales como es el caso de festividades calendáricas y espontáneas (PAC-B32).

\section{Conclusiones}

A base de los resultados empíricos del estudio etnográfico, se aprecia que los pescadores de Playa Carpayo se han constituido debido a la emergencia del restaurante Carpayo (PAC-A3). Posiblemente la aparición de éste, fomentó la presencia de pescadores artesanales locales para abastecer a sus comensales. Asimismo, la colocación de rompeolas desde La Punta hasta Chucuito, es una de las causas principales (PAC-A4) (Figuras 2). Anteriormente, podían pescar libremente por La Punta y Chucuito, pero con la aparición de la isla de Gilligan (PAC-A4) intensifican la restricción de los pescadores artesanales en Playa Carpayo (Figura 3). Respecto al parentesco, estos pescadores son de procedencia local y no local, siendo los primeros de la Provincia Constitucional del Callao (PAC-A1) y lo segundos migrantes, específicamente de la Provincia de Huaral (PAC-A3).

En relación a la perspectiva social de los pescadores de Carpayo, es una labor tradicional practicada a temprana edad (PAC-A1; PAC-A6; PAC-A7), cuyos conocimientos son transmitidos de manera intergeneracional (PAC-B2). De esta manera, los conocimientos empíricos y experienciales asimilados tratan sobre el movimiento de la luna y su influencia en el mar/pesca (PAC-B6), la estacionalidad (PAC-B5), el empleo de redes con tamaños estandarizados para una pesca selectiva y racional (PAC-B7; PAC-B20) y el conocimiento de la etología de los peces (PAC-B3). De igual modo, para incrementar la producción, se utiliza el conocimiento especializado sobre georreferenciación (PAC-B4; PAC-B26) y decodificación de la tabla de oleaje (PAC-B6; PAC-B16).

Sobre el reclutamiento de la tripulación, se realiza a base de la experiencia de los pescadores, debido a que la edad no influye (PAC-B8; PAC-B9); por lo que cuenta con personas que tengan experiencia y estén físicamente preparados para la faena. A pesar de ello, son independientes y no están asimilados a un gremio (PAC-B10).

Con respecto al aspecto económico, los niveles de producción a pequeña escala se ven reflejados en sus medios de producción como es la dimensión de sus embarcaciones (PAC-B12), la capacidad de carga (PACB11), el número de redes (PAC-B13), el tamaño de sus mallas (PAC-B14) y el uso limitado y estacional de hielo (PAC-B18). Por su parte, priorizan una pesca selectiva en vez de colectiva, debido a que buscan la captura de corvinas, cojinovas y fortunos, por su alto valor en el mercado, pero en temporadas bajas pescan alternativamente la lorna. Todo ello es vendido en el muelle y en el mercado a través de los intermediarios, pero en ocasiones ellos mismos trasladan los productos marítimos para abastecer a los comerciantes.

En relación a la conciencia ambiental, los pescadores de Playa Carpayo tienen grosso modo conocimientos sobre el cuidado del ciclo de reproducción de los recursos hidrobiológicos, mediante el uso de redes no tan pequeñas o ciegas que permitan capturas de especies que hayan realizado su primer desove (PAC-B20; PACB21). No obstante, reafirman que la perdida de redes en el fondo marino por atacamiento es una cuestión natural de la propia pesca (PAC-B25; PAC-B26; PAC-B27). También, consideran que debería existir un ente fiscalizador sobre todo ello (PAC-B22; PAC-A8). Por su parte, ambos rechazan la contaminación ambiental, a partir del desmonte de basura en el litoral (PAC-B28) y advierten el alto grado de contaminación y descuido de las playas por parte de autoridades (PAC-B24). En consecuencia, no existe una conciencia ambiental en los pescadores de Playa Carpayo, a pesar que tienen las condiciones necesarias para poder evitar estas malas prácticas, dado que no son ignorantes sobre la temática. 
Respecto al nivel educativo de los pescadores de Playa Carpayo es muy variado, ya que oscila desde el nivel Básico hasta el Superior (PAC-B30), y en función al plano ideológico, se compartes ciertas festividades como el Día del Pescador y el Día del Señor del Mar.

En líneas generales, se demuestra que los pescadores artesanales de Playa Carpayo comparten rasgos generales con otras comunidades de pescadores, pero a su vez contemplan ciertas particularidades circunscritas en el aspecto histórico. Además, cabe destacar que estos resultados son preliminares y deberán ser contrastados con futuras investigaciones con la finalidad de tener una visión más detallada y a profundidad sobre los pescadores citados.

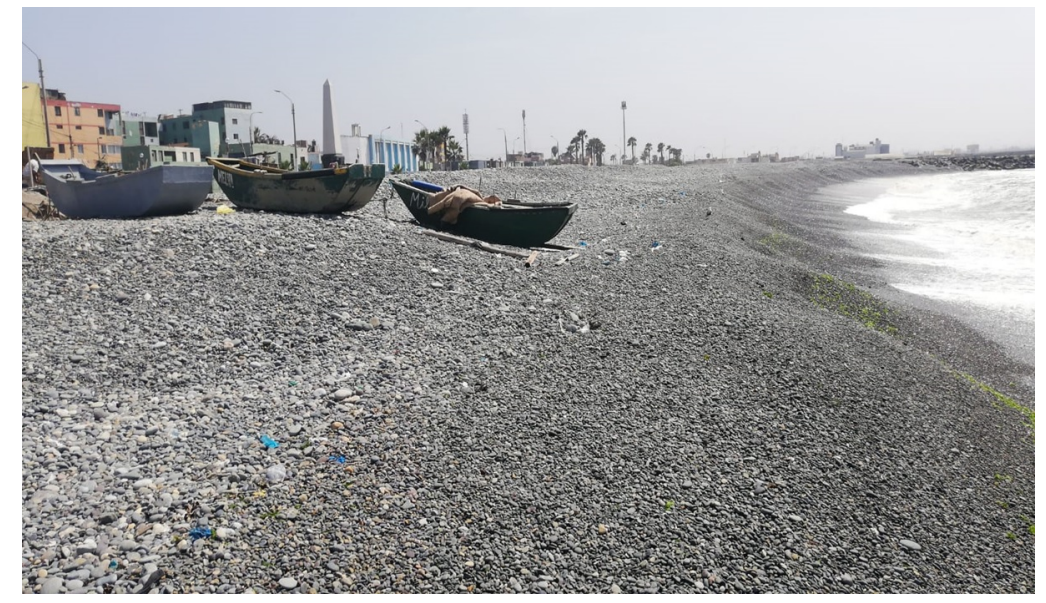

FIGURA 1

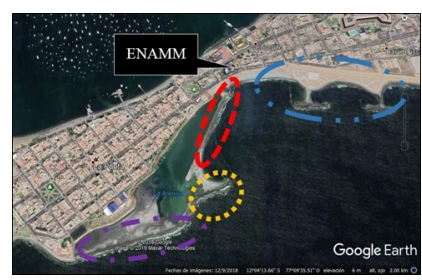

FIGURA 2

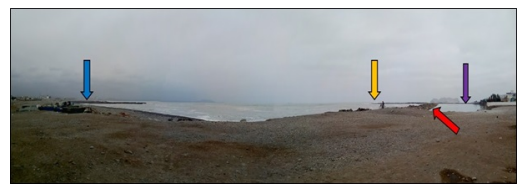

FIGURA 3

\section{REFERENCIAS BIBLIOGRÁFICAS}

Agencia Española de Cooperación Internacional. (2003). Diagnóstico socio-económico de los pescadores artesanales de Ilo y Tácna. Ilo: Centro de Educación, Organización y Promoción de Desarrollo. Disponible en: http:// aecid.pe/images/documentos/2006/doc.20.pdf

Álvarez-Gayou, J. (2009). Cómo hacer investigación cualitativa. Fundamentos y metodología. México D.F.: Paidós.

Cruz, O. (2007). El trabajo de campo como descubrimiento y creación. En M. Minayo, S. Ferreira, O. Cruz, \& R. Gomes, Investigación social: Teoría, método y creatividad (M. Urquía, Trad., págs.41-52). Bueno Aires: Lugar Editorial.

Decreto Supremo No012. (14 de marzo de 2001). Aprueban el Reglamento de la Ley General de Pesca. Ministerio de Producción. Disponible en: https://www.produce.gob.pe/landing/pescayconsumoresponsable/ley-general-depesca.pdf 
Decreto Supremo No05. (25 de agosto de 2012). Modifican reglamento de ordenamiento pesquero del recurso anchoveta y anchoveta blanca, establecen zonas de reserva para consumo humano directo y régimen excepcional. Ministerio de Producción. Disponible en: https://busquedas.elperuano.pe/normaslegales/modificanreglamento-de-ordenamiento-pesquero-del-recurso-an-decreto-supremo-n-005-2012-produce-833209-5/

Galarza, E.\& Kámiche, J. (2015). Pesca artesanal: oportunidades para el desarrollo regional. Lima: Universidad del Pacífico. doi:http://dx.doi.org/10.21678/978-9972-57-342-2

Ley N²5977, Ley General de la Pesca. (21 de diciembre de 1992). En Diario Oficial El Peruano.

McGoodwin, J. (2002). Comprender las culturas de las comunidades pesqueras: clave para la ordenación pesquera y la seguridad alimentaria. Roma: FAO. Disponible en: http://www.fao.org/3/y1290s/y1290s00.pdf

Medicina, J. (2014). Pesca artesanal en el Perú. Ingeniería Industrial, 32: 27-58.

Neira, P. (2005). Las comunidades de pescadores artesanales frente a la modernización: el caso de Caleta Queule (Tesis de Pregrado). Universidad de Chile. Disponible en http://repositorio.uchile.cl/handle/2250/106477

Vallaeys, F. (2016). Introducción a la Responsabilidad Social Universitaria RSU. Barranquilla: Universidad Simón Bolívar. Disponible en: http://unionursula.org/wp-content/uploads/2016/11/Libro-intrpduccion-a-larsu-francois-vallaeys.pdf

\section{Anexo}

\section{Diálogo de la entrevista}

\section{Entrevista: $45 \mathrm{~m}$}

Pescador 1: PAC-A

Pescador 2: PAC-B

Ítem 1: ¿Cómo se establecieron en la Playa Carpayo y de dónde vinieron los pescadores?

Yo tengo 73 años. Yo siempre he pescado acá desde muy niño. Yo vivía aquí entre contralmirante Vidal y Carrillo Albornoz jera sano pe! Yo me venía toda la playa pescado cuando no había rompeolas (PAC-A1).

En la punta donde comen los choncholines, ahí había unos rieles donde había casas bien bonitas y en el mismo balneario había un muelle, donde uno se cambia y se bañaba en la Punta, el tranvía llegaba hasta ahí (PAC-A2).

El problema que ha habido acá, es cuando hicieron el restaurante Carpayo y de ahí vino un Señor y se dedicó a la pesca y de ahí vinieron otros, uno llamado El Brujo, otro un Huachano, de ahí comenzaron hacer sus embarcaciones chiquitas llamadas hasta ahora zapatitos. Yo pescaba de aquí haya y la Sra. Minaya que hasta ahora vive en la esquina puede contarle más de esto (PAC-A3).

Hay un amigo llamado Tarzán que vive en la Siberia, antes esto era un estadio y el mar se salía y todo se inundaba y pasaba todo hasta el Cantolao. De ahí se inició las obras del rompeolas de la Punta y ya la gente se juntó en el rompeolas que hicieron en el Carpayo y ahora último salió el Gilligan, antes era un hueco, yo venía y pasaba de un rompeolas a otro para la pesca. Era bien metido al agua (PAC-A4).

La problemática ahora es que se ha cerrado y la poca de gente de acá que vino a vender a Carpayo, se ha quedado en esta zona. Ya no lo dejan entrar a los de Carpayo para las pozas, los han votado para acá atrás no más (PAC-A5).

Yo tuve mi embarcación cuando mi hija vivía en la Punta, actualmente ya no vive, era para mi nieto, pero no querían que los deje ahí pero mi hija habló con el alcalde y me permitieron dejarla, y estuvo buen tiempo (PAC-A6).

Ítem 2: ¿Desde los cuantos años se dedicó a la actividad pesquera?

Yo salí a pescar desde muy pequeño tendría 8 años, mi papá me llevaba a pasear como se había separado de mi mamá, en su bote alto salía a pescar (PAC-A7). 
Ítem 3: ¿Cómo transfieren las prácticas a sus miembros?

Las prácticas que usamos son sencillas, no hay mucho para enseñar solo se tienen que aplicar bien (PAC$\mathrm{B} 1)$.

Ítem 4: ¿Cómo adquirieron sus experiencias?

La primera vez que salí a pescar tenía 6 años, salí a pescar calamares, justo ahora es temporada de calamar, los boliches lo están depredando. Mis habilidades y capacidades están basadas por mis padres, abuelos y mi experiencia en el campo (PAC-B2).

El problema es el siguiente en la pesca del calamar. El calamar viene a desovar y muere, este viene pone sus huevos y muere hace un esfuerzo y protege sus huevos en el fondo del mar. Yo he buceado entre los 13 y 18 años y puedo explicarle esta experiencia, el calamar tiende aferrarse a sus huevos y pierde la vida porque son fáciles de capturarlos y son también atacados por las rayas y guitarras. Los calamares mientras más se junta, más se protegen, [pero] que hace el boliche como son zonas poca profundas de un máximo $20 \mathrm{~m}$ y sus redes son de 30 a 35 metros, llegan hasta el fondo atrapando no solo el calamar sino también los huevos y depredan la especie, el calamar ha entrado ahorita desde hace varios años 8 a 10 años (PAC-B3).

Ítem 5: ¿Qué conocimientos técnicos aplica en la pesca?

Va depender mucho si hay cerrazón, que no se ve nada y no es recomendable navegar, porque ahorita la flota de los barcos mercantes ya no es la de antes, ahora, es más, los barcos pasan continuamente por una línea, por ejemplo, acá estamos en la 77,00, la primera línea está en la 77,20 o 77,22. Si traes tu navegador, estás navegando por esa zona o por esas líneas puedes tener percance, porque esa línea es de barco, otra línea es la 77,50 por esa línea también pasan barcos, son carreteras específicas que usan los barcos para navegar (PAC-B4).

Ítem 6: ¿Cuáles son sus conocimientos empíricos utilizados en un día de pesca?

Nosotros para empezar la semana nos programamos dependiendo del mes, por ejemplo, mes que estamos entrando al invierno, entonces el tipo de pesca normalmente se hace amaneciendo y durante el día hasta más o menos hasta medio día. Si fuese verano, por el tipo de agua que entran hay más especies que sacar, entonces puedes salir a cualquier hora, porque a cualquier hora puedes encontrar, pero en este tiempo no (PAC-B5).

Otro punto es que para la pesca, el atardecer y el amanecer es clave, es la hora punta de los peces y la luna también, en la noche. Ahora dependiendo de lo que vas a pescar hablamos de cortinas redes, las 2 semanas que va a ver luna te benefician bastante, hoy día ejemplo hay luna hasta las 3 am de la mañana, la luna crea un ambiente de calma, aunque también hay lunas vienteras pero en su mayoría la luna vienen con calma, así el mar está movido que tenga un oleaje que esté en niveles 10,11,12 que es un nivel alto, la luna te va permitir hacer capturas porque hay poco viento, que pasa cuando hay luna y las redes caen dentro del agua ya y cuando no está la luna presente hay una ardentía, se le denomina así porque parece como si ardiera, la especie ve la ardentía y se da la vuelta, ya no ingresan a la red, en cambio cuando hay luna disipa la ardentía, entonces la pone ciega, nosotros decimos está ciego el agua entonces los peces no ven la red y se meten (PAC-B6).

Para estos tiempos nos programamos para salir más o menos 3 am la mañana, porque la mar en estos tiempo va estar en niveles de 10,11,12 y con movimientos anómalos que llegan 16, 17 de movida u oleaje entonces uno tiene que programarse que para este tiempo se tiene las redes de fondo, puede ser en medidas pequeñas de $21 / 2$ pulgadas y las más grandes que son de 5 a 6 pulgadas que son para especies como corvinas, chitas y las más pequeñas para extraer productos que es más de pan llevar que es la Lorna, cabinzas especies más pequeñas. Si fuesen los meses de enero o partir de noviembre, diciembre que ya el sol está un poquito fuerte ya la red son de flote porque las especies de fondo se disipan y levanta especies sobre agua que son la cachema, la lisa, la Chauchilla todo ese tipo de especies que están a 2 a 3 metros debajo de la zona de flote (PAC-B7).

Ítem 7: ¿Ustedes cuando arman la tripulación de la nave que reglas utilizan?

Yo personalmente tengo una lanchita, me encargo primeramente que la gente no sea muy bohemia por decirlo de alguna manera, gente que por el mismo ambiente que estamos más o menos nos conocemos a las personas que te puedan acompañar, tu escoges en función de eso (PAC-B8). 
No influye la edad, yo trabajo por ejemplo con Walter, él tiene 67 años y el hombre está fuerte, hace más que uno de 35 o 40 años y prefiero llevarlo a él que a un muchacho que no responda (PAC-B9).

Ítem 8: ¿Ustedes trabajan con un gremio o son independientes?

Soy independiente, no pertenezco específicamente a la ARUPAC del Callao (PAC-B10).

Ítem 9: ¿De qué capacidad son sus botes?

Parece chiquito, pero llegan a cargar 20 cajas, un total de $400 \mathrm{~kg}$, cargan con dificultad (PAC-B11).

Ítem 10: Qué tamaño tiene tu embarcación?

Hasta 12 pies (PAC-B12).

Ítem 11: ¿Cuántas redes llevan a bordo?

Pueden llevar en promedio de 4 a 5 redes (PAC-B13).

Ítem 12: ¿Cuáles son las medidas de la red?

La mayoría pesca con trasmallos malla de 4 pulgadas, sirve para capturar lenguado, chita, corvina, es una pesca selectiva (PAC-B14).

Ítem 13: ¿Cuáles son las especies más capturadas?

A la espalda de la Isla San Lorenzo que es una zona difícil, pero muy buena zona de pesca llegue a pescar unos 350 kilos de corvina, otro día 20 fortunos (PAC-B15).

Ítem: 14: ¿Cómo es un día bueno de pesca?

Esto dependerá de la especie, por ejemplo si pescamos una especie buena como la corvina, no baja de 25 soles las grandes, el problema es que esta especie no se puede pescar todos los días, uno tiene que revisar la tabla de mareas y la tabla de oleajes, normalmente la pescamos con oleajes sobre 16 y 17 oleajes extremos y solamente usas 50 a $100 \mathrm{~m}$ de red para ponerlo en sitios que por experiencia pasan por ahí, entonces el aparejo de pesca se ponen al alba, ahora está amaneciendo a las 6 am o algo mas, entonces tienes que ponerlo 5:30 am máxima, antes de que comience a rayar el día (PAC-B16).

Ítem 15: ¿Ha tenido temporadas donde la pesca es cero?

Este mes es difícil, no ha venido el viento, julio y agosto son los meses de bajada. Entonces nosotros tenemos aparejos de pesca para este tipo situación. La lorna es la especie alternativa considerada la mala hierba del mar, aunque ya no es tan mala hierba (PAC-B17).

Ítem16: ¿Cuando salen a la faena de pesca llevan hielo?

En verano llevamos un cooler con hielo, ya $\mathrm{q}$ a veces trabajamos de noche para conservar la especie. Si es grande se envuelve en papel periódico o con papel de bolsa de azúcar y hielo. (PAC-B18).

Ítem 17: ¿Dónde comercializan su captura?

Lo vendemos en el mismo muelle o al mercado de Ventanilla, en ocasiones lo llevamos nosotros que son muy pocas u otras ocasiones lo compran en el mismo muelle unas 5 cajas $254 \mathrm{~kg}$ cada una, son personas que llevan para restaurante, de las ventas sacamos una parte para la pesca” (PAC-B19).

Ítem 18: ¿Ustedes cómo se dan cuenta que no están depredando?

Tu depredas siempre y cuando estas matando especies pequeñas, ya que tú no tienes que matarlo en reproducción si uno evitar matarlo en reproducción, entonces vas esperar el otro año que haya lo mismo porque se ha reproducido la especie, pero si usamos una cocada pequeña una malla ciega lo vas a matar en pleno crecimiento y no vas a dejar que se reproduzca, entonces tienes que matarlo con un tamaño de malla que te permita que ya haya hecho su primer desove, entonces la idea es no trabajar con redes que maten especies juveniles. Por ejemplo una malla $2 \frac{1}{2}$ te mata especies más grandecitas, pero hay excepciones que se hacen para pesca específicas, uno puede trabajar con $21 / 8$ por ejemplo para lisa voladora, que lo encontramos entre 40 y 50 millas afuera, para extraerlo nosotros no necesitamos que este pececito meta toda su cabeza porque si mete toda la cabeza tienes que pasarlo y bota la gónada y te llena toda la red con gónada y te la malogra, solo necesitamos que ponga la parte delantera la boca nada más entonces solo la desenganchas, es una excepción para extraer esta especie. Pero si esta red lo metes a esta zona matas las crías porque las especies son pequeñas 
$21 / 8$ pulgadas lo que está pasando ahora con los boliches que están sacando peladilla (anchoveta juvenil) (PAC-B20).

Cuando matas especies pequeñas, imagínate una especie que pesa unos 60, 80 a 100 gr imagine que se llega a reproducir matan 10tn 20 toneladas imagínense que llegan a reproducir sacarían 30, 40 toneladas. (PACB21).

Ítem 19: ¿Cuál es su percepción sobre pesca artesanal irracional?

No hay un control a fondo en la pesca de las especies pequeñas, que se embarque una persona y revisar la bolsa y ver que no cumplen con las medidas reglamentarias. De esa manera se puede controlar la pesca de anchoveta. En la pesca de las especies pequeñas también si hubiera una persona con la autoridad suficiente que indique que vacíe la bolsa, tienen que hacerlo. (PAC-B22).

En el mar las especies forman una cadena alimenticia las especies vienen para alimentarse y reproducirse. (PAC-B23).

El mar a nivel de todo el litoral ha sufrido una depredación única y sigue en mi opinión, el que controlaba todo esto era Banchero Rossi, él sí, por eso éramos unos de los primeros, lamentablemente las autoridades que ha habido no han hecho nada y ya desde años atrás, antes metíamos nuestras redes y salían peces grandes. (PAC-A8).

Ítem 20: ¿Cuál es la percepción del plástico y de los residuos tangibles en la faena?

Las playas de ventanilla son una fábrica de plásticos, esta tiene la particularidad que cuando uno va navegando por ejemplo 2, 3 de la tarde y encuentras un fileo de plásticos, como una carretera de plásticos de 50 metros de ancho aproximadamente, hasta donde dé tus ojos, y de un momento a otro se hunde y si este fileo está cerca a la costa y botas tu red ahí, te das cuenta que se ha hundido y la red se llena de plásticos y tardamos horas en sacarla, realmente no sé cómo llamar a este acontecimiento. La parte más afectada es la Playa de Ventanilla Barlovento, el Rincón de Ventanilla, que se puede llenar hasta 2 mercantes. (PAC-B24).

Anteriormente esta playa era rica, era puro arena había muy muy incluso había de todo hasta camarones que venían en la desembocadura del río Chillón ahora ya no, todo llega contaminado (PAC-A9)

Ítem 21: ¿Alguna vez han perdido su red y qué es lo que hacen?

Si he perdido la red, y he dejado redes y esto se da porque nuestra pesca es selectiva, entonces voy buscando diferentes áreas y estas son zonas rocosas, se va buscando con la red y choca ya que nosotros no trabajamos con sonda para darnos cuenta que choca con el piso, lo que hago es marco con el GPS, y a la próxima vez abro y ya no trio la red en esa zona. La red la dejó, la red no puedo recuperarla porque si lo hago solo sale el cabo, ya que la red se queda atorada. En temporadas de marea, los buzos bajan y recuperan parte de la red, algunos pueden venderlos como chatarra y se recupera algo del material, pero en su mayoría están en mal estado. (PAC-B25).

Actualmente, un armador Lucho le ha sucedido que todas sus redes se han quedado fuera, debido al movimiento de las corrientes, el viento cambia su dirección de sur a norte y esto es terrible y eso provoca la pérdida de la red. También se pierde cuando uno entra en zonas de rompientes, es algo natural, ya que cada uno sabe con la experiencia que si viene la doble llena la red (PAC-B26).

Ítem 22: ¿Hay alguna manera de evitar que se pierda la red?

No hay manera ya q es algo natural (PAC-B27).

Ítem 23: ¿Qué hacen con los residuos que llevan a bordo?

Lo recomendable es guardarlo, yo he trabajado en lanchas grandes, he sido cocinero de 350 toneladas de anchoveta y todos los días nos obligaban a desembarcar la basura, aquí personalmente yo lo hago, pero no puedo opinar por el resto (PAC-B28).

Ítem 24: ¿Han observado algunas especies con parásitos?

Esta semana hemos capturado unos 600 kilos de lorna y lo que me dio bastante pena es que unas seis sí tenían en su vientre y su cabeza enredadas con plástico salen con carachas, le comen la piel y ya no se consume. (PAC-B29).

Ítem 25: ¿Cuál es tu nivel de estudios y como se refleja en la faena? 
Yo estuve llevando la carrera de ingeniería pesquera, la deje un tiempo, pero la estoy retomando. (PACB30).

Sinceramente en la pesca tu no necesitas tener un documento o saber algo, simplemente tener ganas de salir, ya en el trayecto lo aprendes, es fácil salir a la pesca. Ahora de que tú seas bueno en la pesca, ya es muy diferente, porque pueden haber 50 o 100, pero no se desempeñan de la misma forma que te desempeñas tú. Entonces, ahí haces la diferencia, en este punto tú te puedes comparar con los demás, porque uno hace una pesca selectiva y no colectiva, no pesca lo que hacen todos, haces una pesca selectiva, más de tamaño, más de buena especie. Por el tiempo, me he encontrado con gente que comparten conmigo y hemos tenido buenas pescas, hermosas pescas y ves la diferencia. Traer unas cojinovas, grandes fortunos, pardos son especies raras verlas en el mercado, son especies caras, difícil que lleguen al mercado porque no son abundantes. (PAC-B31).

Ítem 26: ¿Ustedes celebran algunas festividades vinculadas a la pesca artesanal?

Normalmente se celebra el día del pescado, el 29 de junio. Después unas fiestas de creyentes como la del Sr. del Mar u otras ya que el pescador festeja cuando quiere. (PAC-B32).

Los autores otorgan el permiso a compartir y usar su trabajo manteniendo la autoría del mismo.

CC BY-NC 